\section{PML risk stratification using anti-JCV antibody index and L-selectin}

\author{
Nicholas Schwab, Tilman Schneider-Hohendorf, Béatrice Pignolet, Michela Spadaro, \\ Dennis Görlich, Ingrid Meinl, Susanne Windhagen, Björn Tackenberg, Johanna Breuer, \\ Ester Cantó, Tania Kümpfel, Reinhard Hohlfeld, Volker Siffrin, Felix Luessi, \\ Anita Posevitz-Fejfár, Xavier Montalban, Sven G Meuth, Frauke Zipp, Ralf Gold, \\ Renaud A Du Pasquier, Christoph Kleinschnitz, Annett Jacobi, Manuel Comabella, \\ Antonio Bertolotto, David Brassat and Heinz Wiendl
}

\section{Abstract}

Background: Natalizumab treatment is associated with progressive multifocal leukoencephalopathy (PML) development. Treatment duration, prior immunosuppressant use, and JCV serostatus are currently used for risk stratification, but PML incidence stays high. Anti-JCV antibody index and L-selectin (CD62L) have been proposed as additional risk stratification parameters.

Objective: This study aimed at verifying and integrating both parameters into one algorithm for risk stratification.

Methods: Multicentric, international cohorts of natalizumab-treated MS patients were assessed for JCV index (1921 control patients and nine pre-PML patients) and CD62L (1410 control patients and 17 prePML patients).

Results: CD62L values correlate with JCV serostatus, as well as JCV index values. Low CD62L in natalizumab-treated patients was confirmed and validated as a biomarker for PML risk with the risk factor "CD62L low" increasing a patient's relative risk 55-fold $(p<0.0001)$. Validation efforts established 86\% sensitivity/91\% specificity for CD62L and 100\% sensitivity/59\% specificity for JCV index as predictors of PML. Using both parameters identified $1.9 \%$ of natalizumab-treated patients in the reference center as the risk group.

Conclusions: Both JCV index and CD62L have merit for risk stratification and share a potential biological relationship with implications for general PML etiology. A risk algorithm incorporating both biomarkers could strongly reduce PML incidence.

Keywords: Natalizumab, PML, L-selectin, CD62L, JCV index, risk stratification

Date received: 18 July 2015; revised: 18 August 2015; accepted: 25 August 2015

\section{Introduction}

Natalizumab was approved for the treatment of active relapsing-remitting multiple sclerosis (RRMS) in 2006. ${ }^{1,2}$ Its main complication has been the risk of developing John Cunningham virus (JCV)-mediated ${ }^{3}$ progressive multifocal leukoencephalopathy (PML), with 566 cases through June 3, 2015.4,5 Patients are currently stratified using three parameters: prior immuno-suppressant use/immune suppression (IS), duration of natalizumab treatment, and presence of antibodies against the PML-inducing
JCV. ${ }^{6}$ Unfortunately, the calculations of future PML incidences and, therefore, risks drawn from data gathered until February 2012, turned out to be underestimated. While Bloomgren and colleagues worked with an incidence of 2.13 (95\% confidence interval $1.85-2.44),{ }^{6}$ the incidence has increased since then, with the most current incidence being almost twice as high with 3.96 (95\% confidence interval 3.644.30). ${ }^{4}$ This suggests that current risk stratification algorithms are not effective enough in preventing the development of new PML cases. ${ }^{7}$ This, of course,
Multiple Sclerosis Journal 2016, Vol. 22(8) 1048-1060

DOI: $10.1177 /$ 1352458515607651

(C) The Author(s), 2015 Reprints and permissions http://www.sagepub.co.uk/ journalsPermissions.nav

Correspondence to: Nicholas Schwab Heinz Wiendl Department of Neurology, University of Münster, Albert-Schweitzer-Campus-1, Building A01, 48149 Münster, Germany. nicholas.schwab@ ukmuenster.de heinz.wiendl@ukmuenster. de

N.S., T.S.H., and B.P. contributed equally to this work.

D.B. and H.W. contributed equally to this work.

Nicholas Schwab Tilman SchneiderHohendorf

Johanna Breuer Anita Posevitz-Fejfár Sven G Meuth Heinz Wiendl Department of Neurology, University of Münster, Germany

Béatrice Pignolet Pole des Neurosciences Centre Hospitalier Universitaire Toulouse, CPTP INSERM UMR 1043 et Université de Toulouse, UPS, France

Michela Spadaro Antonio Bertolotto Clinical Neurobiology Unit, Regional Referring Multiple Sclerosis Centre (CRESM), Neuroscience Institute Cavalieri Ottolenghi (NICO), University Hospital San Luigi Gonzaga, Orbassano, Italy

Dennis Görlich Institute of Biostatistics and Clinical Research, University of Münster, Germany Ingrid Meinl Tania Kümpfe Reinhard Hohlfeld Institute for Clinical Neuroimmunology, LudwigMaximilians-University Munich and Munich Cluster Systems Neurology (SyNergy), Germany 
is also due to the reluctance of patients to deal with their estimated PML risk, the superior efficacy of natalizumab ${ }^{8}$ and the lack of alternative treatments in many cases. ${ }^{9}$ Recently, two new numerical parameters for improving individual risk stratification have been proposed: anti-JCV antibody titers represented as a JCV index value ${ }^{10}$ and $\mathrm{L}$-selectin $(\mathrm{CD} 62 \mathrm{~L})^{11,12}$ as a possible biomarker to indicate individual risk of developing PML under therapy with natalizumab or during human immunodeficiency virus (HIV) infection. ${ }^{13}$ We integrated these numerical risk parameters for natalizumab-associated PML and present verification and validation cohorts for CD62L. Integration of JCV index and CD62L into risk stratification algorithms has the potential to significantly reduce the risk of PML during treatment with natalizumab.

\section{Material and methods}

\section{Patients and biomaterials}

Peripheral blood mononuclear cell (PBMC) samples from 1410 RRMS patients and serum samples from 1921 RRMS patients, both alongside natalizumab therapy, in addition to 17 patients (PBMC) and nine patients (serum), who later developed natalizumabassociated PML (pre-PML), were assessed (Supplementary Figure 1). Furthermore, healthy donors' (HD, $n=90)$ ), systemic lupus erythematosus (SLE, $n=62$ ), HIV $(n=32)$, lymphopenia-associated PML $(n=1)$, SLE-associated PML (SLE-PML; $n=1$ ), HIV-associated PML (HIV-PML; $n=12$ ), and acute natalizumab-associated PML (Nat-PML; $n=$ 19) samples were included. The study was approved by the local ethics committee (University of Muenster: Ethik-Kommission der Ärztekammer Westfalen-Lippe und der Medizinischen Fakultät der Westfälischen Wilhelms-Universität, registration number: 2010-245-f-S; University of Turin: Ethic approval number 7777 (March 25, 2013); University of Barcelona: ethics approval PR(AG)32/2008; University of Toulouse, Comite ethique du sud ouest et outre mer II: 2-09-02) and informed written consent was obtained from all participants. This study was performed according to the Declaration of Helsinki.

\section{Anti-JCV antibody status and-index value}

Sera samples were processed and analyzed by Unilabs (Copenhagen, Denmark) with the second-generation enzyme-linked immunosorbent assay (ELISA kit STRATIFY JCVTM DxSelect ${ }^{\text {TM14 }}$ (\#EL1950, Focus Diagnostics, Cypress, CA, USA) according to the manufacturer's instructions. ${ }^{10}$

\section{CD62L (L-selectin) assessment}

Cryopreserved PBMCs were used as biomaterials. Because of the retrospective assessments of the verification cohorts, standard operating procedures (SOPs) for cryopreservation varied. Thawing and staining protocols, however, were harmonized between centers and applied as published previously; the validation cohorts were cryopreserved and assessed strictly according to the previously published SOPs. ${ }^{11}$ The flow cytometry gating strategy is shown in Figure 1. There is an inherent phenomenon of reduced viability/cellular integrity $(\mathrm{CI} ; \%$ of large $\mathrm{CD}^{+} \mathrm{T}$ cells within $\mathrm{CD} 4^{+} \mathrm{T}$ cells; ${ }^{15,16}$ these smaller lymphocytes do not express CD62L and are propidium iodide (PI) positive in previously cryopreserved lymphocytes. Dead cells were, therefore, excluded from the analysis. The retrospective samples were analyzed in a blinded fashion. After the CD62L results were obtained, the patients were grouped into "control" and "pre-PML." The CD62L results of the prospective samples were reported back to physicians in a blinded fashion over the years. If patients later developed PML, these patients' samples were then grouped into "pre-PML."

\section{Statistics}

Continuous variables such as age and natalizumab cycles are characterized by mean and standard deviation. Categorical variables, e.g. prior IS or JCV seropositivity are described by absolute and relative frequencies. Univariate correlations are estimated by Spearman's correlation coefficient. Data are visualized as scatterplots and supplemented by linear regression lines. Fisher's exact test was used when calculating relative PML risks. $P$ values are considered significant if $\leqslant 0.05$. No adjustment for multiplicity was performed. Statistical analyses were carried out using Prism (Version 5, GraphPad, San Diego, CA, USA) and SPSS (Version 22.0, IBM, Armonk, NY, USA).

\section{Results}

\section{CD62L: Multicentric verification and validation}

To confirm the previously published results of the discovery cohort, ${ }^{11} \mathrm{CD} 62 \mathrm{~L}$ values were assessed in several patient cohorts independently (Supplementary Figure 1). There were two parallel approaches: 1) Already cryopreserved PBMC samples were assessed retrospectively as verification cohorts (BioNAT, assessed in Toulouse, France; Barcelona, assessed in Barcelona, Spain; Marburg, assessed in Muenster, Germany), and as 2) new PBMC samples were
Susanne Windhagen

Department of Neurology,

Clinics Osnabrück, Germany

Björn Tackenberg

Department of Neurology,

Philipps University and

University Clinics Gießen

and Marburg, Germany

Ester Cantó

Xavier Montalban

Manuel Comabella

Servei de Neurologia-

Neuroimmunologia, Centre

d'Esclerosi Múltiple de

Catalunya (Cemcat), Institut

de Recerca Vall d'Hebron

(VHIR), Hospital Universitar

Vall d'Hebron, Universitat

Autònoma de Barcelona,

Spain

Volker Siffrin

Felix Luessi

Frauke Zipp

Department of Neurology,

University of Mainz,

Germany

Ralf Gold

Department of Neurology, Ruhr University Bochum, Germany

Renaud A Du Pasquier Divisions of Immunology and Allergy and of Neurology, Centre Hospitalier Universitaire Vaudois, Switzerland

Christoph Kleinschnitz Department of Neurology, University of Würzburg, Germany

Annett Jacobi

Division of Rheumatology and Clinical Immunology, University of Münster, Germany/Division of Rheumatology and Clinical Immunology, Brandenburg Medical School, Neuruppin Germany

David Brassat

Pole des Neurosciences Centre Hospitalier Universitaire Toulouse CPTP INSERM UMR 1043 et Université de Toulouse, UPS, France/David Brassat also represents the BioNAT study group. 

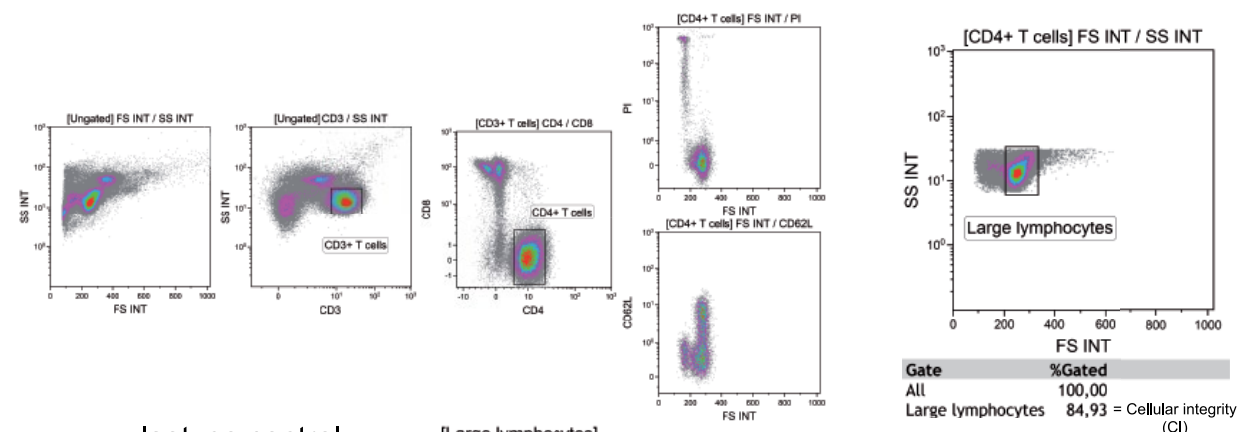

Isotype control
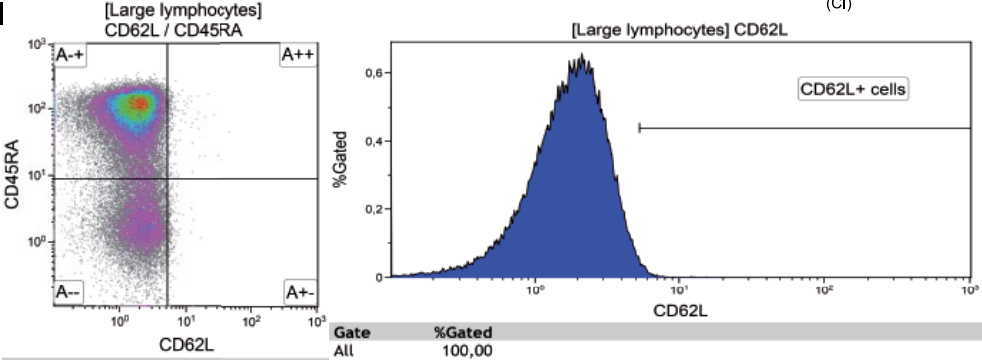

anti-CD62L

(a)

Healthy donor

High cellular integrity

(b)

Healthy donor

Low cellular integrity

(c)

Natalizumab patient Low Cellular Integrity

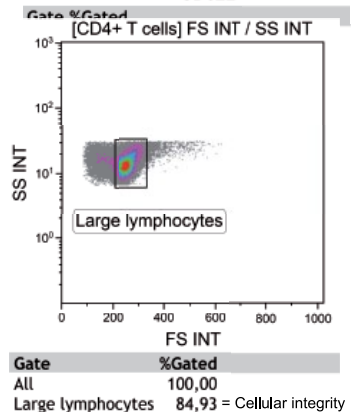

$\begin{array}{lr}\text { Gate } & \% \text { Gated } \\ \text { All } & 100,00 \\ \text { CD62L+ cells } & 0,71\end{array}$

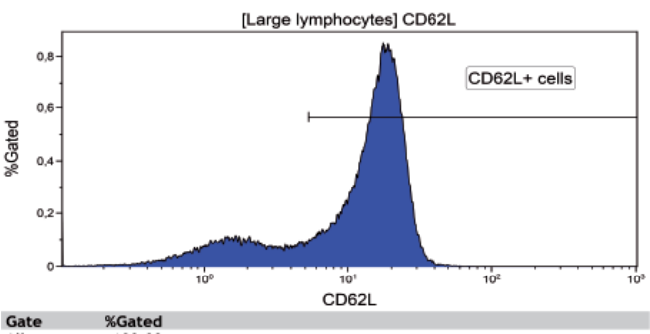

Large lymphocytes $84,93=$ Celluar integily
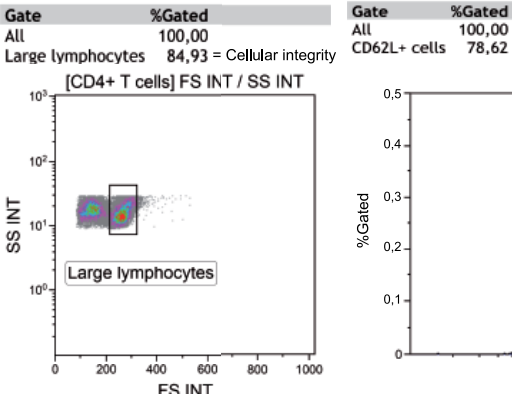

[Large lymphocytes] CD62L

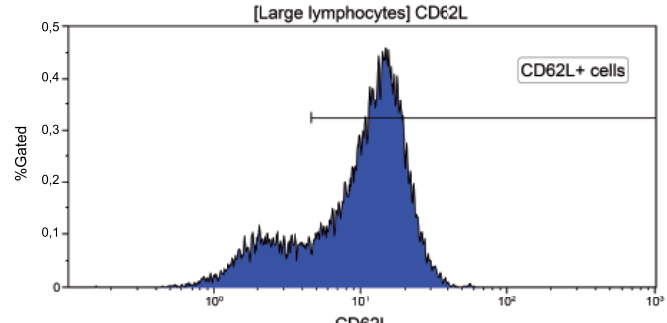

\%Gated
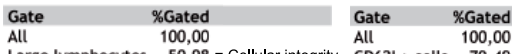

CD62L

$\begin{array}{llll}\text { All } & 100,00 & \text { All } & 100,00 \\ \text { Large lymphocytes } & 50,98=\text { Cellular integrity } & C D 62 \text { + cells } & \\ 79,49 & \end{array}$

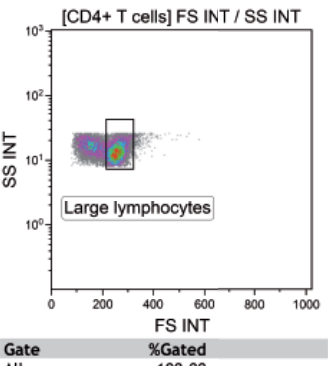

[Large lymphocytes] CD62L

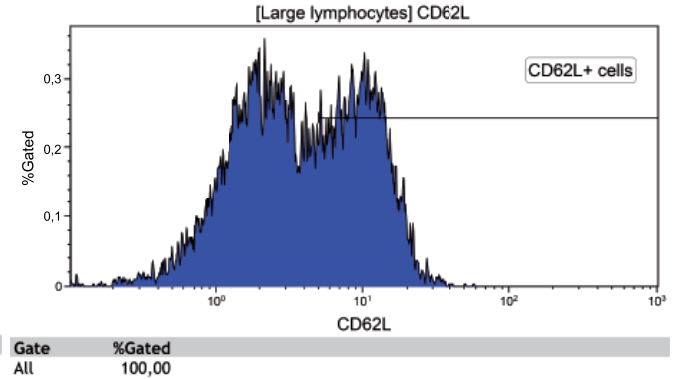

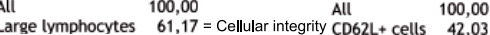

Figure 1. Flow cytometry gating.

PBMC samples are gated on $\mathrm{CD}^{+}$, then $\mathrm{CD}^{+} \mathrm{CD}^{-}$, then on large lymphocytes. Small lymphocytes are propidium iodide/PI and CD62 $\mathrm{L}^{-}$. CD62 $\mathrm{L}^{+}$cells are subsequently determined according to the isotype control. (a) Healthy donor sample with high CI. (b) Healthy donor sample with low CI. (c) Natalizumab-treated patient with low CI. PBMC: peripheral blood mononuclear cell; CI: cellular integrity. 
collected prospectively according to the previously published rigorous $\mathrm{SOPs}^{11}$ as validation cohorts (Turin, Italy; Mainz, Germany (both assessed locally); Wuerzburg and Muenster, Germany (both assessed in Muenster)). Of note, eight of the 17 pre-PML patients have been described previously. ${ }^{11}$ The retrospectively analyzed verification cohorts of natalizumab-treated MS patients ( $n=497$ controls +10 pre-PML patients) presented with a highly significant CD62L difference between control patients $($ mean $=23.36+-14.37)$ and patients who later developed PML (pre-PML, mean = $5.88+-4.34 ; p<0.0001)$. Additionally, the samples, which were prospectively cryopreserved in five locations especially for cellular CD62L assessment, showed a mean for controls of $45.85+-13.59(n=$ $913)$, which again was significantly higher than the values of patients who later developed PML $(n=7$; mean $=17.11+-13.67 ; p=0.0001)$. A threshold for PML risk was calculated using the exhaustive $\mathrm{CHi}$ squared Automatic Interaction Detection (CHAID) algorithm with a significance level for splitting of $1 \%$, resulting in a threshold of 10.42 for the retrospective verification cohorts, leaving 93 of 497 measured control patients $(18.7 \%)$ below the threshold. In the prospective validation cohorts, the threshold could be raised to 27.95 because of the rigorously applied SOPs for biomaterial shipping, processing, and measurement, resulting in much higher specificity. This threshold set 85 of 913 control patients $(9.3 \%)$ as "CD62L low." If several PBMC samples of a single patient were available, the lowest measured value was used for the cross-sectional analysis to depict the most accurate result of a long period of observation (up to five years for some patients) (Figure 2(a)). Statistical analysis set the relative PML risk for CD62L-low patients in the validation cohorts at 54.55-fold ( $p<$ 0.0001) when compared to CD62L-normal patients. The sensitivity in the validation cohort was $85.7 \%$ (95\% confidence interval 42.0-99.3) (six of seven patients who later developed PML were measured as being CD62L low) and the specificity was $90.79 \%$ (95\% confidence interval 88.5-92.5).

\section{Assessment of CD62L in different patient cohorts associated with PML risk and of the JCV index in a large German cohort of natalizumab-treated MS patients}

CD62L was also assessed in several other disease entities with PML risk. Interestingly, occurrence of acute-PML also coincided with low CD62L values. This could be shown in SLE (one of one CD62L-low PML patients), HIV (11/12 CD62L-low PML patients), lymphopenia (one of one CD62L-low PML patients), and most important, natalizumab-associated
PML (14/19 CD62L-low PML patients) in the acute stage of PML (Figure 3(a)). JCV index (risk threshold $>0.9)$ showed a specificity of $58.77 \%(100 \%-41.23 \%)$ (Figure 3(b)), and JCV index was available for nine pre-PML patients. The threshold of 0.9 was chosen for maximum sensitivity, as it was shown that only $4.4 \%$ of patients presented with values $<0.9$ before developing PML compared to $13.2 \%$ of patients presenting with values $<1.5 .{ }^{17}$ Five of these patients were non-IS and all their index values were $>1.5$ (Figure $3(\mathrm{~b})$ ); i.e. $100 \%$ sensitivity of the JCV index in the five eligible $\mathrm{JCV}+$ patients. However, one of these nine patients was JCV negative six months prior to PML diagnosis, leading to a sensitivity of the JCV serostatus in our cohort of $89 \%$.

\section{Correlation between the anti-JCV index value and CD62L expression}

There was no influence of CI on the expression of CD62L on viable lymphocytes in healthy controls, SLE patients or MS patients without natalizumab treatment. Interestingly, there was a strong correlation between $\mathrm{CI}$ and expression of CD62L on remaining viable cells, especially visible in natalizumab-treated MS patients (Spearman rho $=0.378 ; p<0.0001$ ) (Figure 4(a)). The potential influence of CI on the JCV index value of the same blood sample was evaluated in parallel. In patients treated with natalizumab, samples with lower JCV index showed higher CI ( $p=$ 0.039) (Figure 4(b)). Consequently, as CI influences the expression of CD62L, as well as being associated with the risk classification of the JCV index according to a threshold of 0.9 , natalizumab-treated patients with low JCV index cross-sectionally had higher CD62L values $(n=121$; mean $=52.89)$ and vice versa $(n=122$; mean $=49.43 ; p=0.042)$ (Figure 4(c) $)$. CD62L values did not differ between pre-PML patients and controls in freshly isolated PBMCs (Figure 4(d)), only in previously cryopreserved PMBCs. Chemically induced shedding of CD62L (e.g. by phorbol 12-myristate 13-acetate (PMA)) in fresh PBMCs did not distinguish pre-PML samples from controls (data not shown). Importantly, it became evident that the lower the CD62L value a patient had, the higher the probability of being $\mathrm{JCV}^{+}$or seroconverting to JCV seropositivity (Figure 5(a)), leading to 35 of 37 CD62L low patients of the Muenster cohort being $\mathrm{JCV}+$ in their most recent serum sample. This also fit with the observation that CD62L and JCV index correlated directly (Spearman rho $=-0.128 ; p=$ 0.014 ) (Figure 5B). In this cross-sectional analysis of 370 patients in the reference center, seven $(1.9 \%)$ presented with both risk factors: JCV index $>0.9$ and CD62L value $<27.95$. 
(a)
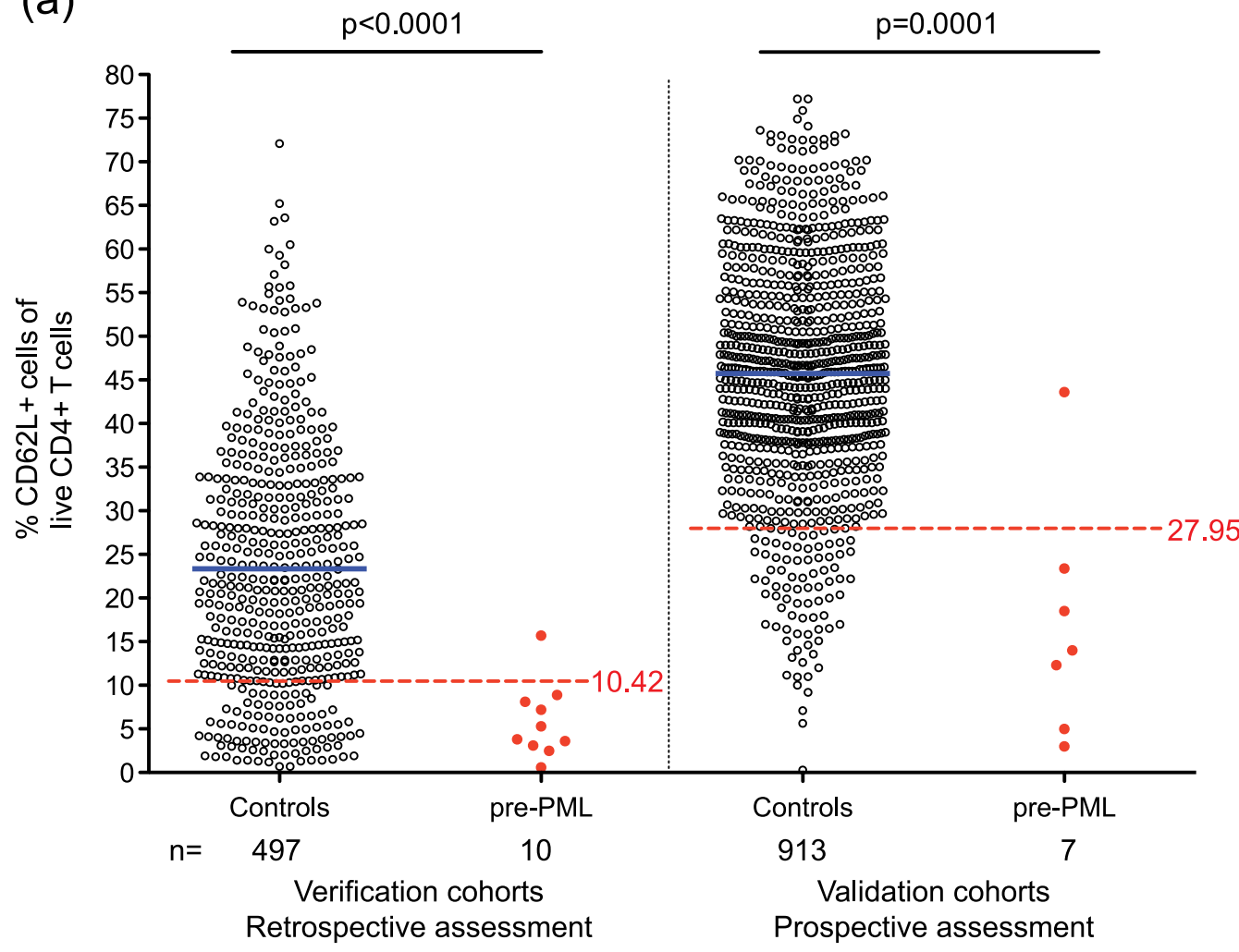

(b)

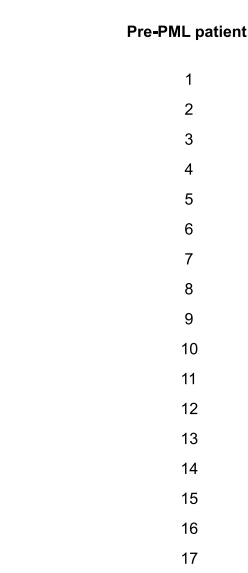

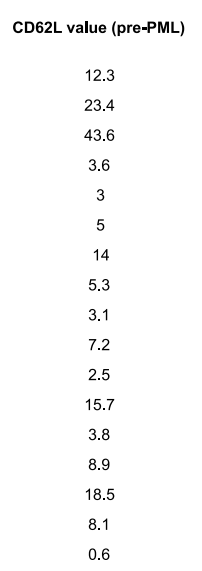

$\begin{gathered}\text { CD62L assessment } \\ \text { at infusion \# }\end{gathered}$
56
30
71
15
12
12
18
25
27
25
25
24
41
24
38
15
19
$\mathbf{2 8 . 1}$

PML diagnosis
after infusion \#

Duration between CD62L diagnosis (months) 10

10
19

66

49
82

82

45
29

24

38

50

44

47

45

43
83

45

45

39

31

Mean

Prospective assessment

Figure 2. CD62L: Multicentric verification and validation.

(a) CD62L values (\% CD62 $\mathrm{L}^{+}$cells of live $\mathrm{CD}^{+} \mathrm{T}$ cells; mean: blue lines) of the retrospective verification cohorts (BioNAT, Barcelona, and Marburg): 497 controls: mean $=23.36+-14.37,10$ pre-PML patients: mean $=5.88+-4.34$. Threshold: 10.42 . Sensitivity: $90 \%$, specificity: $81 \%$. CD62L values of the prospective validation cohorts (Turin, Mainz, Wuerzburg, and Muenster): 913 controls: mean $=45.85+-13.59$, seven pre-PML patients: mean $=17.11+-13.67$. Threshold: 27.95 . Sensitivity: $86 \%$, specificity: $91 \%$. Thresholds (dotted red lines) are calculated based on statistical algorithms for optimal specificity/sensitivity (exhaustive CHAID). PML: progressive multifocal leukoencephalopathy; CHAID: CHi-squared Automatic Interaction Detection.

(b) Given are pre-PML patient numbers, CD62L values, natalizumab infusion number at the time point of CD62L assessment, natalizumab infusion number at the time point of PML diagnosis, duration between the CD62L assessment and PML diagnosis, JCV index value, whether there was prior immune suppression, and whether this patient was published previously. Of note, a different sample aliquot was assessed in previously published patients. ${ }^{11}$ PML: progressive multifocal leukoencephalopathy; JCV: John Cunningham virus. 


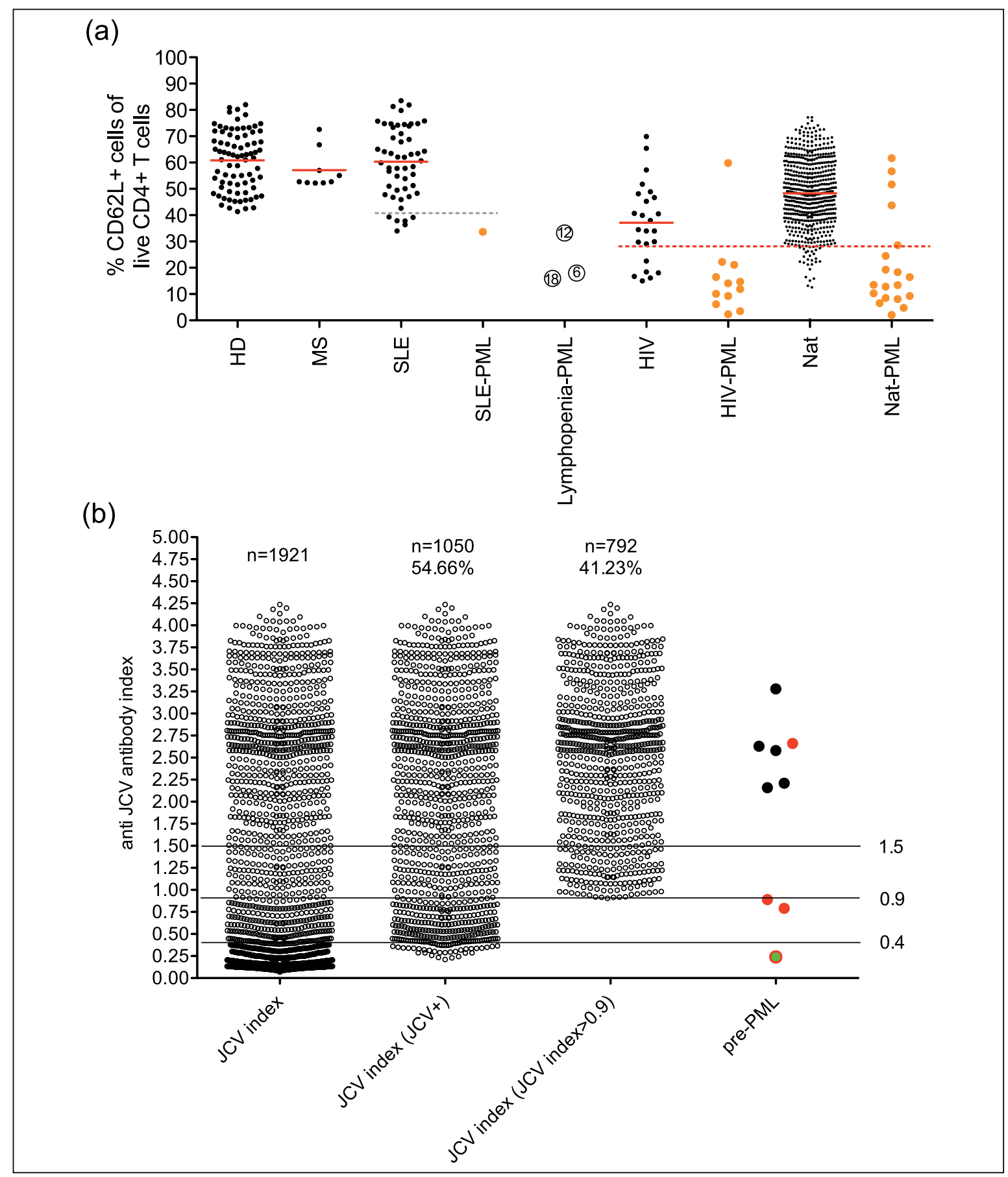

Figure 3. Assessment of CD62L in different associations of PML and of JCV index in a large cohort of German patients treated with natalizumab.

(a) Shown are the CD62L values of the patient cohorts (\% CD62L $\mathrm{L}^{+}$cells of live CD4+ $\mathrm{T}$ cells): $\mathrm{HD}(n=79)$, SLE $(n=52)$, SLE-PML $(n=1)$, HIV $(n=24)$, HIV-PML $(n=12)$, lymphopenia-associated PML $(n=1 ; 3$ time points, numbers are "months post-PML diagnosis"), MS patients not treated with natalizumab (MS, $n=9$ ), MS patients treated with natalizumab and monitored in Muenster (Nat, $n=605$ ), and patients suffering from acute natalizumab-associated PML (Nat-PML, $n=19$ ). The red dotted line indicates the validated threshold (27.95), which sets $4 \%$ of the patients assessed in Muenster as CD62L low. The gray line is a tentatively suggested threshold for SLE patients at 40.6, which would set 11.5\% of SLE patients as being CD62L low in their respective cohort. Statistical threshold determination was not possible with one SLE-PML case. PML: progressive multifocal leukoencephalopathy; HD: healthy donors; SLE: systemic lupus erythematosus; HIV: human immunodeficiency virus; MS: multiple sclerosis.

(b) JCV index of 1921 natalizumab-treated patients grouped according to being JCV+ (54.66\%) and the index risk threshold $0.9(41.23 \%)$, resulting in a specificity for the JCV index in our cohort of 58.77\% (100\%-41.23\%). Pre-PML patients are shown in black (non-immune suppressed) or red (prior immune suppression). The patient, who was JCV negative before PML onset, is shown in green with a red border (indicating prior immune suppression). The black lines show the thresholds of 0.4 (serostatus JCV+), 0.9 (lower PML risk threshold) and 1.5 (higher PML risk threshold). JCV: John Cunningham virus; PML: progressive multifocal leukoencephalopathy. 
(a)

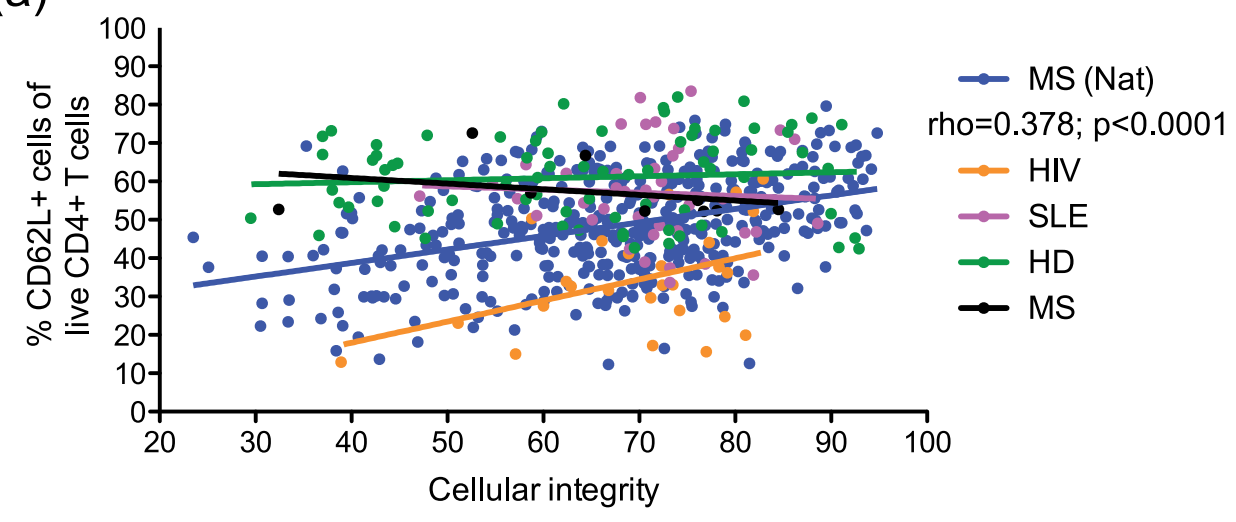

(b)

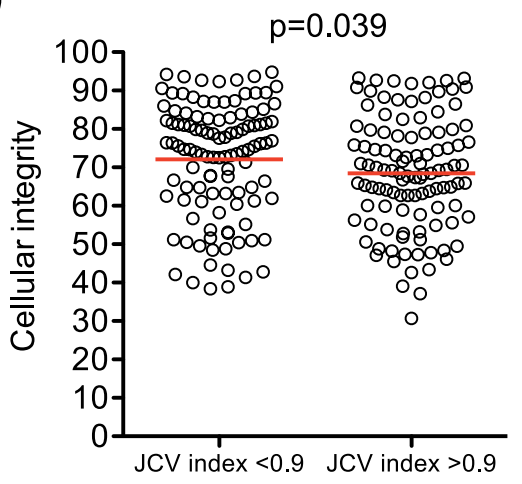

(c)

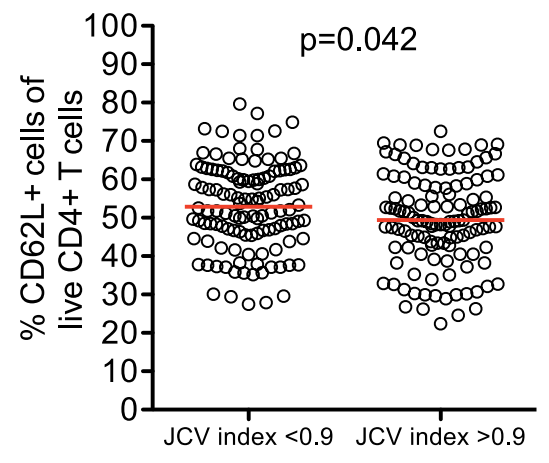

(d)

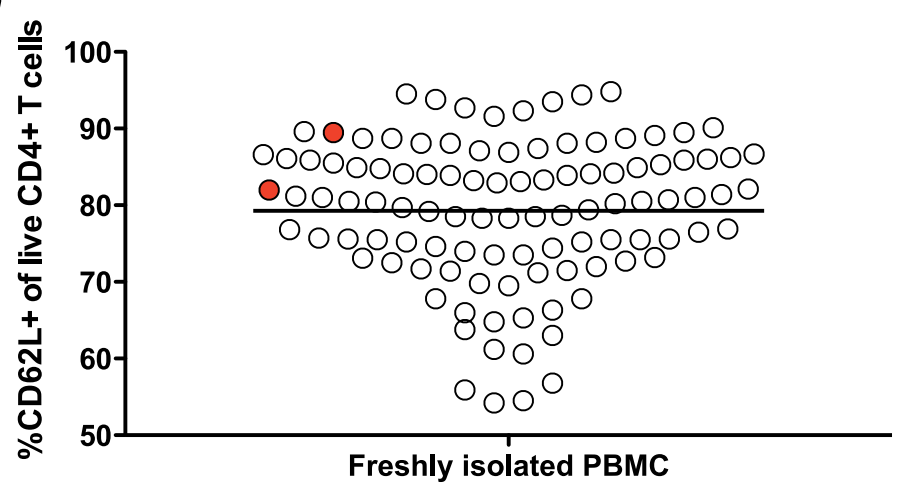

Figure 4. Correlation between cellular integrity (CI), JCV index and CD62L.

(a) Correlation between CI and CD62L value in the cohorts of Figure 2(b) (Nat $n=423$; slope $=0.353$; Spearman rho $=0.378$; $p<0.0001)$. (b) CI of natalizumab-treated MS patients grouped according to JCV index value $(<0.9: n=111$; and $>0.9: n=109)$.

(c) CD62L value of natalizumab-treated MS patients grouped according to JCV index value (<0.9: $n=121$; and $>0.9: n=122)$.

(d) Shown are 105 patients (103 non-PML controls, two pre-PML patients) under long-term treatment with natalizumab. Stainings (as delineated in Figure 1) were performed on freshly isolated PBMCs. Samples of pre-PML patients (red) do not show reduced CD62L values in fresh blood. JCV: John Cunningham virus; PML: progressive multifocal leukoencephalopathy; MS: multiple sclerosis; PBMC: peripheral blood mononuclear cells.

\section{Discussion}

The fact that current PML incidences were underestimated by previous studies ${ }^{4,6}$ strengthens the need for efficient risk stratification parameters (especially for $\mathrm{JCV}+$ patients), as using algorithms based on mainly the JCV serostatus has been only modestly successful in reducing the occurrence of new PML cases. ${ }^{7}$ Some biomarker efforts have yielded promising results in the recent literature. ${ }^{18,19}$ Large prospective studies will have to determine their usefulness, applicability, 


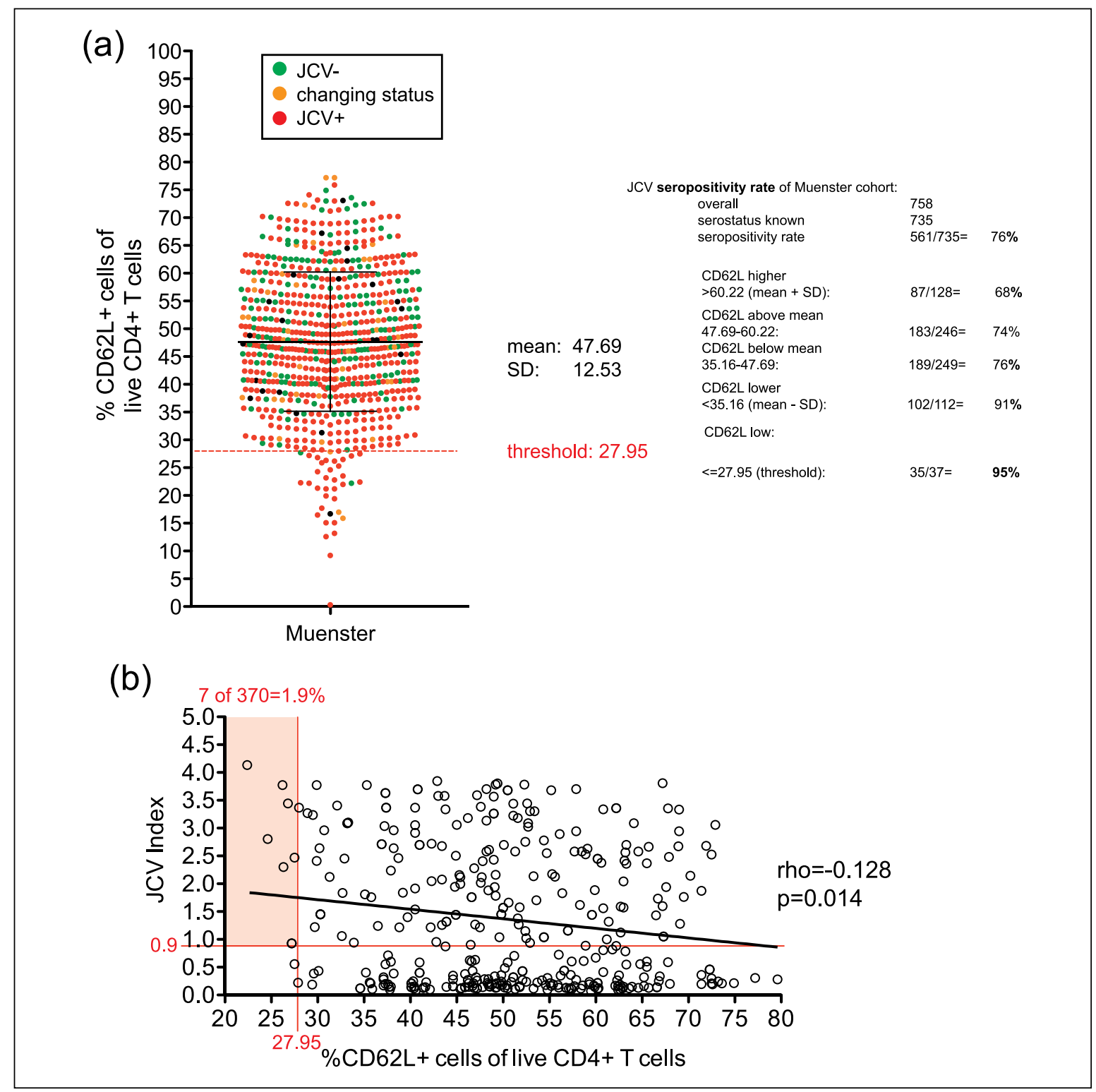

Figure 5. Correlation between CD62L and JCV serostatus, as well as JCV index.

(a) CD62L values of the Muenster cohort $(n=758)$. If patients were measured several times during the validation period, the lowest measured CD62L value is shown to account for the longest possible period of observation/longitudinal stability. $\mathrm{JCV}^{-}$patients are shown in green, $\mathrm{JCV}^{+}$patients in red, patients who changed serostatus during the period of observation in orange, and patients with unknown JCV status in black. With lower CD62L values, the percentage of $\mathrm{JCV}^{+}$patients rises steadily; 35 of 37 (95\%) CD62L-low patients are $\mathrm{JCV}^{+}$. (b) Correlation between CD62L and JCV index in natalizumab-treated MS patients $(n=370$; slope $=-0.01721 \pm 0.00543$; $p=0.0017$; Spearman rho $=-0.128 ; p=0.014)$. Red lines depict the thresholds for JCV index (0.9) and CD62L (27.95), and the red quadrant indicates the patients of this cohort set at high risk to develop PML in the future (seven of $370=1.9 \%$ ). JCV: John Cunningham virus; PML: progressive multifocal leukoencephalopathy; MS: multiple sclerosis.

connection to other risk factors, and how they can be incorporated into daily clinical practice. We focused on the two numerical biomarkers potentially relevant for individual PML risk stratification during natalizumab therapy: JCV index and CD62L.

The different CD62L mean values of the verification and validation cohorts show that it is important to ship, process, and measure samples strictly according to the published $\mathrm{SOPs}^{11}$ to ensure optimal assay specificity. Within the verification and validation cohorts, the sensitivity of the biomarker CD62L was $90 \%$ and $86 \%$, respectively (nine of 10 and six of seven prePML patients were CD62L low at least once). This is in agreement with the largest PML cohort (HIV), in whom CD62L showed a sensitivity of $92 \%$ during 


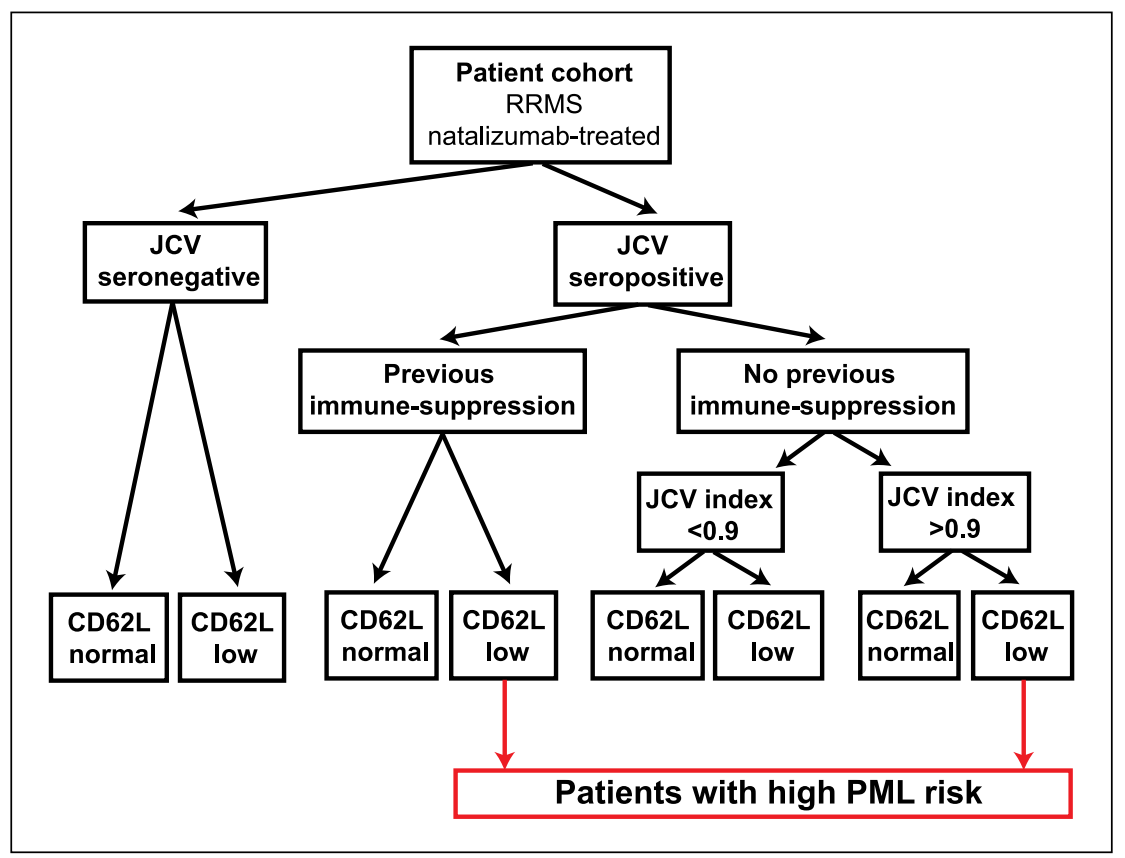

Figure 6. Suggested PML risk stratification algorithm.

Suggested risk stratification algorithm integrating JCV index and L-selectin. This suggested algorithm shows how to incorporate JCV index value and L-selectin into clinical practice. Statistically, adherence to this scheme should avoid most future PML cases by considering a change in treatment in a few patients. In short, natalizumab-treated patients should be tested for JCV serology and CD62L. $\mathrm{JCV}^{+} \mathrm{CD} 62 \mathrm{~L}-$ low patients should consider treatment alternatives, unless they are not immune suppressed and their JCV index is $<0.9$. PML: progressive multifocal leukoencephalopathy; JCV: John Cunningham virus; RRMS: relapsing-remitting multiple sclerosis.

acute PML. Importantly, these results were achieved using random assessment intervals and with a mean duration of 19.3 months between the last CD62L assessment and PML diagnosis and should, therefore, be higher with regular (e.g. bi-yearly) monitoring. Based on the validation cohorts, a proposed risk stratification algorithm would set a small group of MS patients at high risk (CD62L low combined with being $\mathrm{JCV}+$ and a) either IS or b) JCV index $>0.9$ ) (Figure 6). CD62L specificity can be $>91 \%$ with optimal SOPs (e.g. in the Muenster reference center, 96\% of control patients stay above the CD62L threshold over time and only $1.9 \%$ of patients present with both risk factors). While retrospective cohorts are mainly important for reproduction purposes, showing that the assay works per se, standardized prospective cohorts can be used for threshold discussions and daily clinical practice.

Analogous to the JCV index, one single CD62L threshold of 27.95 will not be ideal for every cohort, but also not for every circumstance, as risk stratification is multiparametric and individual. While statistics and distributions set this threshold for optimal sensitivity and specificity during validation, it might be that a patient with other treatment options might decide that, personally, a CD62L value above that threshold is unacceptable, whereas a patient with limited therapeutic alternatives might want to stay on treatment even with a lower value.

Low CD62L values seem to be associated with PML in all constellations we looked at (natalizumab, rituximab, efalizumab, ${ }^{11} \mathrm{HIV},{ }^{13}$ SLE and lymphopenia), leading us to conclude that the biomarker might be generally applicable for PML risk assessment and suggesting a common PML etiology in all of these instances: In total, 46 of 54 patients $(85 \%)$ from all PML associations and stages presented with the risk factor CD62L low.

In this study, we were able to demonstrate that known PML risk parameters such as natalizumab treatment duration, JCV index, and $\mathrm{CD} 62 \mathrm{~L}$ value are related in various ways. This suggests that these risk factors are indicators for a "status" of the immune system, which would make a patient vulnerable specifically to the development of PML. This connection is also strongly supported by the correlation between JCV index and CD62L value in the same blood sample, leading to the finding that $96 \%$ of patients who presented with even one CD62L-low PBMC sample, were $\mathrm{JCV}^{+}$in their most recent serum sample. As a further confirmation, it was recently shown that in addition to the cellular 
form, soluble CD62L also correlates with JCV index in natalizumab-treated MS patients. ${ }^{20}$

In a similar line of thought it makes sense that in the first 17 months of treatment, when the PML incidence is much lower, none of 69 patients was set as CD62L low. As previous hypotheses concerning JCV titers suggested that higher titers are the result of a higher replication rate of the virus, ${ }^{21-23}$ it is conceivable that a higher replication rate is due to the fact that the lessviable immune cells of this patient are less capable of suppressing viral activity. Consistent with the fact that samples with low CI show less CD62L after thawing, which is a stressful event for the cells, both numerical risk parameters (high JCV index and low CD62L) might potentially be the downstream result of a dysfunctional immune status, albeit assessing different parts of the immune system: humoral and cellular. Remarkably, changes in CI related to CD62L loss on live cells were especially strong in natalizumab-treated patients. Reduced CI leads to lower CD62L levels and higher JCV index values, both associated with enhanced PML risk. The CD62L value should, therefore, really be regarded as a very specific immune cell stress indicator due to the freezing/thawing procedure. This point of view is supported by the fact that in fresh blood stainings, the CD62L value of pre-PML patients is not reduced.

$\mathrm{CD} 62 \mathrm{~L}$ is for several reasons not useful for diagnosing acute PML: First and foremost, the procedure including cryopreservation and waiting periods takes roughly three weeks to perform with rigorous SOPs, rendering any result useless for immediate treatment decisions. Secondly, the sensitivity of the assay in acute PML patients is currently only $74 \%(14 / 19$ acute PML patients were measured as CD62L low), possibly due to the different nature of the PML associations natalizumab versus HIV, which causes $91.7 \%$ of patients to stay CD62L low during acute PML. However, the results from the cohort of patients suffering from acute natalizumab-associated PML strongly support the validity of CD62L as a risk biomarker for PML and also support the chosen thresholds. Of note, one of the acute-PML cases was tested as JCV seronegative six months prior to PML diagnosis, but presented with a low CD62L value at diagnosis.

Recently, the manufacturer of natalizumab (Biogen) investigated CD62L as a potential PML risk biomarker ${ }^{24}$ and could show that eight out of nine pre-PML patients presented with at least one low CD62L value. The ninth pre-PML patient presented as CD62L low only during acute PML. Because of the retrospective nature of this specific study, the mean of control patients and the longitudinal stability of CD62L were reduced, similar to what we observed in our retrospective cohorts. This strongly reduced the specificity of the marker. However, our data clearly show that prospective assessment and rigorous adherence to SOPs for clinical diagnostics solve this problem. The group of Professor Bertolotto (Turin) successfully reproduced the results of the initial study independently by following SOPs and suggests that CD62L might be a valid biomarker for PML risk during natalizumab treatment. ${ }^{25}$

Both numerical biomarkers of PML risk prediction have strengths and weaknesses. The JCV index is determined from serum, which is easy to ship. The index's overall sensitivity is difficult to judge, as a) it does not work for IS patients, who make up a large proportion of PML patients and, therefore, the eligible cohort is reduced and b) there are at least four JCV patients (who, therefore, had an index value of $<0.4$ ) who developed PML, ${ }^{4}$ but these patients are not discussed in the index publication, because the paper describes only $\mathrm{JCV}^{+}$(pre-PML) patients. One of these JCV-seronegative patients is shown in this publication. Based on the currently available data, the sensitivity of the JCV index in anti-JCV antibody-positive patients without prior immunosuppressant use is, therefore, $96 \%(65 / 68)$, based on the 0.9 index cutoff. ${ }^{10,17,26}$ If the JCV-seronegative PML cases are included, the combined sensitivity of the JCV serology will be lower than that. Additionally, its specificity of detecting patients at risk is low at $58.77 \%$. CD62L, on the other hand, is a parameter measured in PBMCs isolated from ethylenediaminetetraacetic acid (EDTA) blood with all its inherent challenges of shipment and storage, but its current sensitivity of $86 \%-88 \%$ and specificity of $91 \%-96 \%$ can be considered very high and it works in patients with prior IS. Moreover, for CD62L, being a dynamic biomarker, we emphasize that patients with one normal value are not free of risk, as they could have been CD62L low earlier. However, close monitoring from the start of therapy on can mitigate this problem and the marker should, therefore, be regarded as a qualitative risk factor, similar to IS: A patient tested as CD62L low even once has a statistically validated 55-fold higher risk of later developing PML. As there are few situations conceivable that might in singular cases lead to false-positive results (e.g. heart attack, ${ }^{24}$ immediate pre-treatment with fingolimod (data not shown), or acute urosepsis ${ }^{27}$ ), physicians should try to assess their patients during periods with low interference by concomitant treatments or afflictions. 
The next step for the implementation of the biomarker could be the setup of standardized and validated laboratories in each country where natalizumab is being prescribed, to allow for a manageable and easy assessment of CD62L in daily clinical practice. This, however, is beyond the scope of an academic center and should be pursued by the pharmaceutical industry.

Ultimately, natalizumab is still one of the most efficacious treatments available today, and risk stratification during treatment with natalizumab is about individual risk perception and tolerance. The systematic inclusion of both JCV index and CD62L could reduce the risk and occurrence of PML up to 10-fold if applied rigorously during risk stratification.

\section{Acknowledgements}

The authors would like to thank Barbara Wrobel, Petra Babucke, Julia Schlingmann, Verena Schütte, Schumina Säuberlich, Anke Schwabe, Hannah Holtkötter, Grigory Gerasimov, Florence Bucciarelli, Lise Scandela and Florence Umuhoza for excellent technical assistance and the patients for their participation in the study. The corresponding authors NS and HW had full access to all of the data in the study and take responsibility for the integrity of the data and the accuracy of the data analysis.

Author contributions are as follows: NS designed and performed research, collected data, analyzed data, and generated funding; TS-H designed and performed research, collected and analyzed data; BP and MS performed research, collected and analyzed data; DG performed statistical analyses; IM, SW and BT collected patient data and helped with patient recruitment; JB and EC performed research, collected and analyzed data. TK, APF and RH collected patient data and helped with patient recruitment; VS, FL and FZ performed research, collected and analyzed data. XM, SGM, RG, CK, RdP and AJ collected patient data and helped with patient recruitment. MC performed research, collected and analyzed data, and generated funding; AB performed research, collected and analyzed data; DB performed research, collected and analyzed data, and generated funding; HW designed research, analyzed data and generated funding; all authors wrote the manuscript.

\section{Conflicts of interest}

The authors BP, DG, APF, AJ, VS, JB and EC have nothing to declare. NS has received honoraria for advisory boards and travel expenses from Biogen Idec. TS-H has received travel expenses compensation from Biogen Idec. IM has received travel expenses from
Bayer-Schering. SW has received honoraria for lecturing and travel expenses for attending meetings from Bayer-Schering, Biogen Idec, Merck-Serono, Novartis, Sanofi-Aventis, and Teva. TK has received travel expenses and personal compensations from Bayer-Healthcare, Teva, Merck-Serono, Novartis, Sanofi-Aventis, and Biogen Idec as well as grant support from Bayer-Schering AG. RG reports scientific grant support, consulting and speaker honoraria from Biogen Idec, Baxter, Bayer-Schering, Chugai, MerckSerono, Novartis, Sanofi-Aventis, Teva, and ZLBBehring. DB has received honoraria for lecturing and travel expenses for attending meetings and boards from Bayer-Schering, Biogen Idec, Merck-Serono, Novartis, Sanofi-Aventis, and Teva. RdP serves on scientific advisory boards for Biogen Idec, MerckSerono, and Novartis; has received funding for travel or speaker honoraria from Biogen Idec, SanofiAventis, Merck-Serono, and Bayer Schering; serves on the editorial boards of the European Journal of Neurology and the Journal of Neurovirology; and receives research support from the Swiss National Foundation, the Swiss Society for Multiple Sclerosis, and the Biaggi Foundation. RH has served on scientific advisory boards for, and received research grant support from, Novartis, Biogen Idec, Bayer-Schering, Merck-Serono, Sanofi-Aventis, Teva, and Genzyme. CK has received honoraria for lecturing, consulting fees, and research support from Bayer Healthcare, Biogen Idec, Boehringer Ingelheim, Biotronik, Bristol Myers Squibb, Eisa, Merck-Serono, Novartis, Sanofi Genzyme, Pfizer, Siemens, and Teva. BT has received honoraria for lecturing; consulting fees from Biogen Idec, Bayer Healthcare, Genzyme, Merck-Serono, Novartis, Sanofi-Aventis, and Teva; and research support from Biogen Idec, Bayer Healthcare, Novartis, Sanofi-Aventis, and Teva. AB has received honoraria for serving on the scientific advisory boards of Almirall, Bayer Healthcare, Biogen Idec, and Sanofi Genzyme and has received speaker honoraria from Biogen Idec, Sanofi Genzyme, Novartis, and Teva. His institution has received grant support from Bayer Healthcare, Biogen Idec, Merck, Novartis, Teva, the Italian MS Society, Associazione Ricerca Biomedica ONLUS, and San Luigi ONLUS. MS has received travel grant and speaker honoraria from Biogen Idec. FZ has received research grants and travel compensation from Teva, Novartis, Merck-Serono, and Bayer Healthcare, as well as consultation funds from Johnson \& Johnson, Novartis, Ono, and Octapharma. FL has received travel grants from Merck-Serono and Teva. $\mathrm{MC}$ has received speaker honoraria from Bayer Schering, Merck-Serono, Biogen Idec, Teva, SanofiAventis, and Novartis; is on the editorial board of the 
Journal of Neuroimmunology and Journal of Clinical Immunology; and has received research support from Red Espanola de Esclerosis Multiple, Ajuts per donar Suport als Grups de Recerca de Catalunya, and the Marie Curie Initial Training Network "UEPHA*MS." HW has received honoraria and consultation fees from Bayer Healthcare, Biogen Idec, Fresenius Medical Care, GlaxoSmithKline, GW Pharmaceuticals, MerckSerono, Novartis Pharma, Sanofi Genzyme, and Teva Pharma. NS, TS-H and HW are co-inventors of a patent related to the use of CD62L in PML risk stratification. SGM has received honoraria for lecturing and travel expenses for attending meetings and has received financial research support from Bayer Healthcare, Bayer Schering, Biogen Idec, Sanofi Genzyme, Merck-Serono, MSD, Novartis, Novo Nordisk, Sanofi-Aventis, and Teva. XM has received compensation for consulting services and speaker honoraria from Neuro-Tec, Novartis, Roche, Sanofi Genzyme, Teva, GeNeuro, Genentech, Merck, EMD, Biogen Idec, Bayer Healthcare, and Almirall.

\section{Funding}

This work was funded by the Deutsche Forschungsgesellschaft (DFG) grant CRC128 to NS, RH, FZ, and HW and Cells-in-Motion Cluster of Excellence (EXC 1003-CiM), the PML consortium to NS and HW, the Kompetenznetz Multiple Sklerose (Competence Network for Multiple Sclerosis) funded by the Federal Ministry of Education and Research (FKZ 01GI1308B 01GI0907) to HW, French Ministry of Health (PHRC 2008-005906-38, ARSEP (French MS Society grant 2009 and 2011)) to DB, and the EU (BEST-MS, FP7, 305477) to DB and HW. The funding agencies had no influence on design and conduct of the study, collection, management, analysis, and interpretation of the data, or preparation, review, or approval of the manuscript.

\section{References}

1. Polman CH, O'Connor PW, Havrdova E, et al. A randomized, placebo-controlled trial of natalizumab for relapsing multiple sclerosis. $N$ Engl J Med 2006; 354: 899-910.

2. Miller DH, Khan OA, Sheremata WA, et al. A controlled trial of natalizumab for relapsing multiple sclerosis. N Engl J Med 2003; 348 : $15-23$.

3. Tan CS and Koralnik IJ. Progressive multifocal leukoencephalopathy and other disorders caused by JC virus: Clinical features and pathogenesis. Lancet Neurol 2010; 9: 425-437.
4. Biogen. Biogen Idec Medical information, https:// medinfo.biogenidec.com/ (2015, accessed 3 June 2015).

5. Calabrese LH, Molloy E and Berger J. Sorting out the risks in progressive multifocal leukoencephalopathy. Nat Rev Rheumatol 2015; 11: 119-123.

6. Bloomgren G, Richman S, Hotermans C, et al. Risk of natalizumab-associated progressive multifocal leukoencephalopathy. N Engl J Med 2012; 366: 1870-1880.

7. Cutter GR and Stüve O. Does risk stratification decrease the risk of natalizumab-associated PML? Where is the evidence? Mult Scler 2014; 20: 13041305.

8. Kalincik T, Horakova D, Spelman T, et al. Switch to natalizumab versus fingolimod in active relapsingremitting multiple sclerosis. Ann Neurol 2015; 77 : 425-435.

9. Capobianco M, di Sapio A, Malentacchi M, et al. No impact of current therapeutic strategies on disease reactivation after natalizumab discontinuation: A comparative analysis of different approaches during the first year of natalizumab discontinuation. Eur $J$ Neurol 2015; 22: 585-587.

10. Plavina T, Subramanyam M, Bloomgren G, et al. Anti-JC virus antibody levels in serum or plasma further define risk of natalizumab-associated progressive multifocal leukoencephalopathy. Ann Neurol 2014; 76: 802-812.

11. Schwab N, Schneider-Hohendorf T, Posevitz V, et al. L-selectin is a possible biomarker for individual PML risk in natalizumab-treated MS patients. Neurology 2013; 81: 865-871.

12. Major EO and Douek DC. Risk factors for rare diseases can be risky to define: PML and natalizumab. Neurology 2013; 81: 858-859.

13. Schneider-Hohendorf T, Philipp K, Husstedt IW, et al. Specific loss of cellular L-selectin on CD4(+) $\mathrm{T}$ cells is associated with progressive multifocal leukoencephalopathy development during HIV infection. AIDS 2014; 28: 793-795.

14. Lee P, Plavina T, Castro A, et al. A second-generation ELISA (STRATIFY JCVTM DxSelect ${ }^{\mathrm{TM}}$ ) for detection of JC virus antibodies in human serum and plasma to support progressive multifocal leukoencephalopathy risk stratification. J Clin Virol 2013; 57: 141-146.

15. Brady HJM. Apoptosis methods and protocols. Totowa, NJ: Humana, 2004.

16. Chrest FJ, Buchholz MA, Kim YH, et al. Identification and quantitation of apoptotic cells following anti-CD3 activation of murine G0 T cells. Cytometry 1993; 14: 883-890. 
Visit SAGE journals online http://msj.sagepub.com

๑SAGEjournals
17. Kuesters G. Anti-JC virus (JCV) antibody index differentiates risk of PML in natalizumab-treated MS patients with no prior immunosuppressant use: An updated analysis. Neurology 2015; 84: AAN Poster P4 031.

18. Meira M, Sievers C, Hoffmann F, et al. MiR-126: A novel route for natalizumab action? Mult Scler 2014; 20: $1363-1370$.

19. Villar LM, Costa-Frossard L, Masterman T, et al. Lipid-specific immunoglobulin $\mathrm{M}$ bands in cerebrospinal fluid are associated with a reduced risk of developing progressive multifocal leukoencephalopathy during treatment with natalizumab. Ann Neurol 2015; 77: 447-457.

20. Basnyat P, Hagman S, Kolasa M, et al. Association between soluble L-selectin and anti-JCV antibodies in natalizumab-treated relapsingremitting MS patients. Mult Scler Relat Disord 2015; 4: 334-338.

21. Bohl DL, Brennan DC, Ryschkewitsch C, et al. $\mathrm{BK}$ virus antibody titers and intensity of infections after renal transplantation. J Clin Virol 2008; 43 : 184-189.
22. Pastrana DV, Brennan DC, Cuburu N, et al. Neutralization serotyping of BK polyomavirus infection in kidney transplant recipients. PLoS Pathog 2012; 8: e1002650.

23. Pastrana DV, Wieland U, Silling S, et al. Positive correlation between Merkel cell polyomavirus viral load and capsid-specific antibody titer. Med Microbiol Immunol 2012; 201: 17-23.

24. Lieberman L. CD62L (L-selectin) is not a reliable biomarker for predicting risk of PML in natalizumabtreated MS patients. Neurology 2015; 84: AAN Poster P4 0.39.

25. Spadaro M, Caldano M, Marnetto F, et al. Natalizumab treatment reduces L-selectin (CD62L) in CD4(+) T cells. J Neuroinflammation 2015; 12: 146.

26. Gorelik L, Lerner M, Bixler S, et al. Anti-JC virus antibodies: Implications for PML risk stratification. Ann Neurol 2010; 68: 295-303.

27. Jackson LA, Drevets DA, Dong ZM, et al. Levels of L-selectin (CD62L) on human leukocytes in disseminated cryptococcosis with and without associated HIV-1 infection. J Infect Dis 2005; 191: 1361-1367. 